\title{
Desarrollo de la nueva guía canadiense de diseño y gestión de infraestructura vial 2011: un resumen del estado de la práctica canadiense
}

\section{Development of the new 2011 canadian pavement asset design and management guide: a brief summary of canadian state-of- the-practice}

\author{
Susan L. Tighe*1, Norman W. McLeod*, Marta Juhasz**
}

\author{
* University of Waterloo, CANADA \\ ** Alberta Transportation, CANADA
}

Contribución ICMPA 2011 Contribution ICMPA 2011
PAG. $93-111$

Resumen

En septiembre de 2009, la Asociación de Transporte de Canadá (Transportation Association of Canada, TAC) comenzó a desarrollar la versión 2011 de la Guía de diseño y gestión de infraestructura vial (Pavement Asset Design and Management Guide, PADMG). El proyecto es un fondo de estudios auspiciado por más de veinte organismos públicos canadienses entre los cuales se encuentran los diez Departamentos de Transporte provinciales, varias de las principales ciudades canadienses, diversas asociaciones industriales y el gobierno federal. El proyecto ha sido conducido por un consorcio canadiense compuesto por especialistas y académicos de todo el país. El equipo está compuesto por un variado grupo de profesionales de vasta experiencia en Canadá en los diversos aspectos tratados en esta guía. La nueva Guía PADMG 2011 corresponde a una consolidación del estado de la práctica actual en Canadá en materia de diseño y gestión de pavimentos para ingenieros, autoridades, administradores y técnicos. Además, constituirá una valiosa fuente informativa para estudiantes universitarios y de institutos de formación técnica, tanto en el país como en el exterior. La guía destacará algunos aspectos industriales clave como la sustentabilidad, el cambio climático y las innovaciones. En primer lugar, este trabajo presenta un resumen de los hallazgos encontrados en una encuesta realizada por una parte interesada con el fin de determinar el actual estado de la práctica en Canadá. La información recogida por esa encuesta cubre los principales conocimientos prácticos del diseño y gestión de los pavimentos. La nueva Guía tendrá 15 capítulos que comprenden la discusión sobre los principios de la gestión de infraestructura vial, requerimientos de datos, sustentabilidad, mantenimiento, materiales, diseño de caminos para alto y bajo volumen y análisis del ciclo de vida de los diseños de los pavimentos. El borrador del PADMG 2011 fue aprobado y el proyecto quedará terminado en septiembre de 2011 .

Palabras Clave: Asociación de transporte de Canadá ( TAC), Guia de diseño y gestión de infraestructura vial

Abstract

The development of the new 2011 Transportation Association of Canada (TAC) Pavement Asset Design and Management Guide (PADMG) began in September 2009. The project is a pooled fund study being sponsored by over twenty Canadian public agencies including all ten provincial Canadian Departments of Transportation, several major Canadian cities, several industry associations and the federal government. The effort is being lead by a Canadian consortium team composed of consultants and academics from across Canada. The consortium team is composed of a diverse group of practitioners who have experience across Canada in the various areas of expertise pertinent to this guide. The new 2011 PADMG will be an up-to-date, practical consolidation of Canadian pavement design and management practice for practicing engineers, managers and technicians. It will also be a valuable resource for college and university courses both in Canada and elsewhere. The guide will highlight key industry issues such as sustainability, climate change, and new innovations. The paper first provides a summary of findings from a stakeholder survey to determine current state-of-the-practice in Canada. Information collected in this survey covers the major practices areas in pavement design and management. There will be 15 chapters in the new guide ranging from discussions on the principles of asset management, to data requirements, sustainability, maintenance, materials, low and high volume road design and life cycle analysis of pavement designs. The draft 2011 PADMG has been submitted and the project will be completed by September 2011

Keywords: Transportation association of Canada (TAC), Pavement asset design and management guide (PADMG)

\section{Introducción}

\footnotetext{
1 Autor de correspondencia / Corresponding author: E-mail: sltighe@uwaterloo.ca
}

\section{Introduction}

The development of the new 2011 Transportation Association of Canada (TAC) Pavement Asset Design and Management Guide (PADMG) began in September 2009. 
The project is a pooled fund study being sponsored by over fifteen Canadian public agencies including all ten provincial Canadian Departments of Transportation, several major Canadian cities, several industry associations and the federal government. The effort is being led by a Canadian consortium composed of consultants and academics from across Canada. The consortium is composed of a diverse group of practitioners who have experience across Canada in the various areas of expertise pertinent to the Guide. The new 2011 PADMG is an upto-date, practical consolidation of Canadian pavement design and management practices for practicing engineers, managers and technicians. It will also serve as a valuable resource for education of new engineers and technicians at both the college and university level. It is anticipated as was the case with previous guides that it will be used by international practitioners as a reference. The Guide will highlight key industry issues such as sustainability, climate change, and new innovations.

This type of initiative is not new to the TAC. Three previous guides have been published in 1965, 1977 and 1997. Full use of time invariant material from the 1997 TAC Pavement Design and Management Guide will be made, while outdated information will be removed. New material, concepts and practices, as well as new chapters, will be incorporated in the 2011 PADMG. In essence, the work to be carried out will build on the past but focus on the needs of the future.

\section{Scope or objective of the paper}

The focus of this paper is to address how the 2011 survey outcomes have influenced the process of producing the Guide. The survey helped to identify and subsequently address important issues related to pavement and asset management throughout Canada. This paper shows the 2011 survey results in comparison to the 1993 survey results. This shows the changes that have occurred in the past 18 years. Finally, the paper provides a brief introduction of the topics included in the new 2011 Guide.

\section{Background}

One of the first major tasks in the development of the new 2011 PADMG was to carry out a stakeholder survey to determine the current state-of-the-practice in Canada. The intent of the survey, completed in early 2010, was also to ensure the content of the 2011 Guide is up-to-date and relevant to users. 
This paper is directed at briefly describing the new Guide, summarizing the key findings from the survey, and providing a comparison of the 1997 Guide survey results to the new Guide's survey results (note that the 1997 Guide's survey was done in 1993). Information collected in the $\mathbf{2 0 1 0}$ survey covers the major practice areas in pavement design and management. The comprehensive survey included questions on: network information; pavement and asset management practices; data collection types and methodologies including condition indices; pavement design, construction and materials; pavement preservation, maintenance and rehabilitation practices; new innovations; and select questions on green initiatives and sustainability.

The members of the consortium team, in partnership with the TAC PADMG Project Steering Committee, (PSC) have also identified the prospective chapters in the new Guide. Members of the consortium are currently reviewing reference materials both nationally and internationally to ensure each chapter is relevant and up-to-date. For example, the new Guide will include extensive review of TAC publications, Canadian Technical Asphalt Association (CTAA) proceedings, the Canadian National Guide for Sustainable Municipal Infrastructure (NGSMI), Transportation Research Board (TRB) records, the United States National Highway Cooperative Research Program (NCHRP), Provincial Standards, and other applicable publications. In addition, a list of additional references will be included at the end of each chapter.

The following chapters will be included in the new Guide:

- Chapter 1: Introduction;

- Chapter 2: Principles of Asset Management;

- Chapter 3: Principles of PMS and Implementation;

- Chapter 4: Data Requirements and Collection Methods;

- Chapter 5: Network Level Needs Analysis and Priority Programming;

- Chapter 6: Materials; 
- Chapter 7: Flexible Pavement Design;

- Chapter 8: Rigid Pavement Design;

- Chapter 9: Gravel and Surface Treated Road Design;

- Chapter 10: Maintenance and Rehabilitation Treatments;

- Chapter 11: Life Cycle Analysis of Pavement Designs;

- Chapter 12: Role of Construction;

- Chapter 13: Role of Maintenance;

- Chapter 14: Sustainability in Pavement Engineering and Management; and

- Chapter 15: Issues, Opportunities and Future Prospects.

A detailed schedule for drafts was prepared to ensure the final 2011 PADMG was completed on time in September 2011. Each chapter was prepared by a team of two to three consortium members. Peer review by the consortium project manager and one other consortium member was also carried out on each chapter. A technical editor was also involved in the review of each chapter to ensure the Guide was professional and consistent. Finally each chapter was submitted to the PADMG PSC for their review and comment. The 2011 TAC PADMG was written in a straightforward and consistent manner so that it would be easy to understand. Each chapter was prepared to provide information for the newly graduated engineer or technician starting their career to the senior engineers and managers who require information on complex issues.

In addition, training and workshop materials were prepared by the consortium. This includes a comprehensive set of slides and speaker notes for use during the 2011 TAC Workshop at the annual fall conference and in addition, training across Canada has been planned upon the official release of the 2011 PADMG.

A primer for the 2011 PADMG was also prepared. The primer is directed at summarizing the key pertinent features of the Guide and is also directed at non-technical practitioners, executives and politicians responsible for managing assets. However, it will also serve as a communication tool for technical practitioners when dealing with their various stakeholders. The Primer outlines the key subjects that are addressed in the Guide and briefly describes the current status of Canadian best practices and emerging trends including technology advancements. 
Green transportation technologies, climate change, and other topical technologies are highlighted. The Primer also highlights the importance of various factors involved in the design and management of all pavements in the network including: rural and urban; gravel, surface treated, flexible and rigid; and local, collector, arterial and freeway functional classifications.

\section{Purpose and design of the 1993 and 2010 surveys}

The surveys, carried out in both 1993 and 2010, were directed at acquiring information on current pavement design and management methods and practices and to identifying the preferences of requirements of a representative set of major users of the Guide. The surveys were also similar in format in both years (Tighe, 2009b). Another objective of the surveys was to identify problems or issues currently associated with pavement management systems, what could be done differently, and opportunities for change. Finally, the 2010 survey provided an opportunity for respondents to indicate items they would like to see in the new 2011 Guide.

The 2010 survey, developed by the consortium, was 32 pages in length and quite in-depth and comprehensive. The survey contained 9 major sections which were: General Information; Pavement and Asset Data Requirements, Collection Methods and Database; Network Level Needs Analysis and Priority Programming; Pavement Materials and Drainage; Pavement Design (Structural and Economic Evaluation); Construction; Maintenance and Preservation; Implementation of a Pavement Management and Asset Management System; and Issues, Opportunities and Future Prospects. Within each section there were items related to the information and also items for opinions or additional comments.

\section{Respondents to the survey}

The survey was distributed in January 2010 to organizations across Canada, including cites, provincial, federal and territorial agencies, consulting firms, academic institutions and other organizations associated with the field of pavement design and management. The return rate of the survey was $63 \%$ as opposed to a return rate of $75 \%$ 
for the 1993 survey, although the raw number of surveys returned in 2010 was higher, at 44, than in 1993, at 34. A broader group was surveyed in 2010 when compared to 1993 (Tighe, 2009b). The breakdown of respondents is shown in Table 1 below.

The survey was distributed in January 2010 and all responses were received by March 2010. In total, 44 surveys were received back from 15 cities, 14 federal, provincial and territorial agencies, 8 consultants and supplier agencies, 4 academic institutions and 3 Associations and Public Private Partnerships. It is noteworthy that the survey has also assisted the TAC Asset Management Task Force and the TAC Project Steering Committee on performance issues. It has also been used by the TAC Project Steering Committee on Special Roads.

Tabla 1. Resumen de los encuestados en los años 1993 y 2010

Table 1. Summary of 1993 and 2010 Survey Respondents

\begin{tabular}{|c|c|c|c|}
\hline $\begin{array}{l}\text { Encuesta de } 1993 \\
1993 \text { Survey }\end{array}$ & & $\begin{array}{l}\text { Encuesta de } 2010 \\
2010 \text { Survey }\end{array}$ & \\
\hline $\begin{array}{l}\text { Organismos Federales/Provinciales/Territoriales } \\
\text { Federal/Provincial/Territorial Agencies }\end{array}$ & 14 & $\begin{array}{l}\text { Organismos Federales/Provinciales/Territoriales } \\
\text { Federal/Provincial/Territorial Agencies }\end{array}$ & 14 \\
\hline $\begin{array}{l}\text { Municipalidades } \\
\text { Municipalities }\end{array}$ & 19 & $\begin{array}{l}\text { Municipalidades } \\
\text { Cities and Municipalities }\end{array}$ & 15 \\
\hline \multirow[t]{3}{*}{$\begin{array}{l}\text { Agencias Consultoras y Proveedoras } \\
\text { Consultant and Supplier Agencies }\end{array}$} & 1 & $\begin{array}{l}\text { Agencias Consultoras y Proveedoras } \\
\text { Consultants and Supplier Agencies }\end{array}$ & 8 \\
\hline & & $\begin{array}{l}\text { Instituciones Académicas } \\
\text { Academic Institutions }\end{array}$ & 4 \\
\hline & & $\begin{array}{l}\text { Asociaciones y Sociedades Público- Privadas } \\
\text { Associations and P3's }\end{array}$ & 3 \\
\hline Total & 34 & Total & 44 \\
\hline
\end{tabular}

\section{Summary of 2010 survey findings}

The following is a highly summarized overview of the survey responses, the findings of which will be incorporated into the new Guide. The results are grouped into Cities, Provincial/Federal/Territorial Agencies and Others. The questions that were asked are presented followed by the results. Due to the scope of this paper only selected responses have been summarized, the findings are presented in tables and figures. A more detailed version of the survey summary is also available through TAC (Tighe, 2010a; Tighe and Juhasz, 2010a). The intent of this section is to highlight current practices related to pavement management in Canada. 


\subsection{General Information}

What percentage (of your average annual budget for roads and pavements) is directed to: new pavement construction/reconstruction, rehabilitation, routine maintenance, major maintenance and pavement preservation?

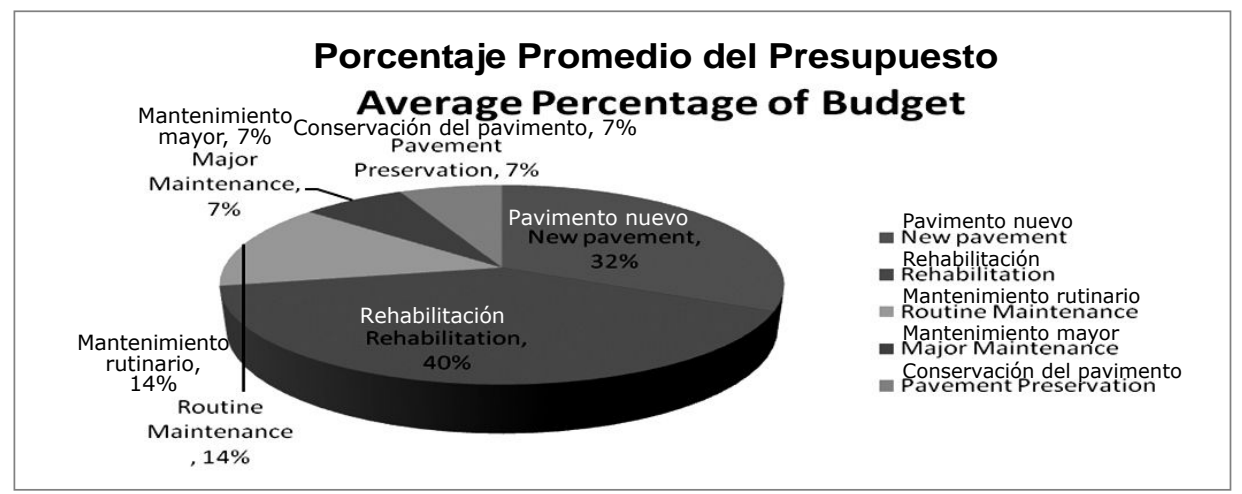

Figura 1. Resumen del gasto en caminos y pavimentos por los organismos federales, provinciales y territoriales Figure 1. Summary of road and pavement spending for federal/provincial/territorial agencies

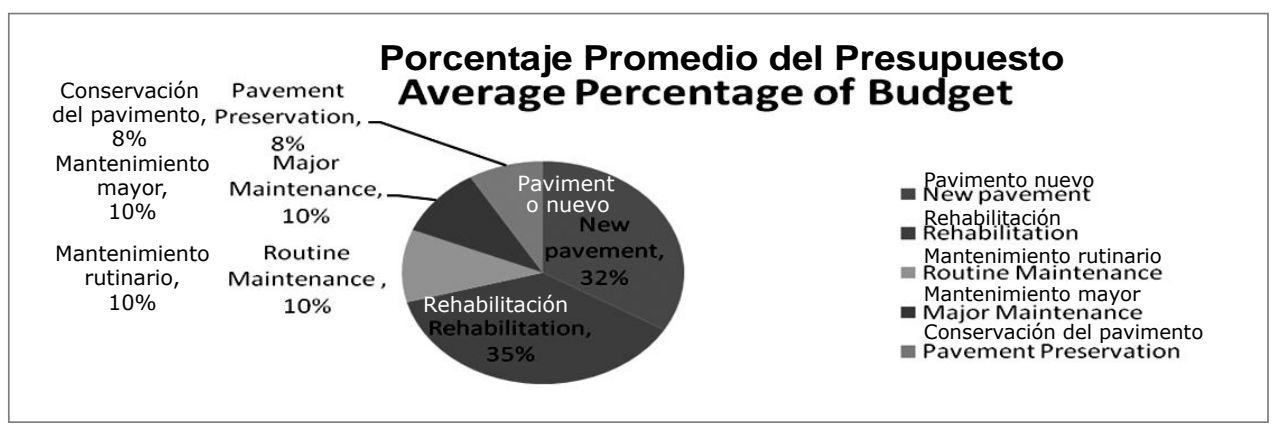

NOTA* Los porcentajes pueden no ser igual al $100 \%$ ya que algunos organismos no entregaron respuestas NOTE* percentages may not equal $100 \%$ as some agencies did not provide answers

Figura 2. Resumen del gasto en caminos y pavimentos por las municipalidades encuestadas Figure 2. Summary of road and pavement spending for cities surveyed

Approximately what percentages of pavements are: gravel, surface treated, flexible (asphalt), rigid and composite?

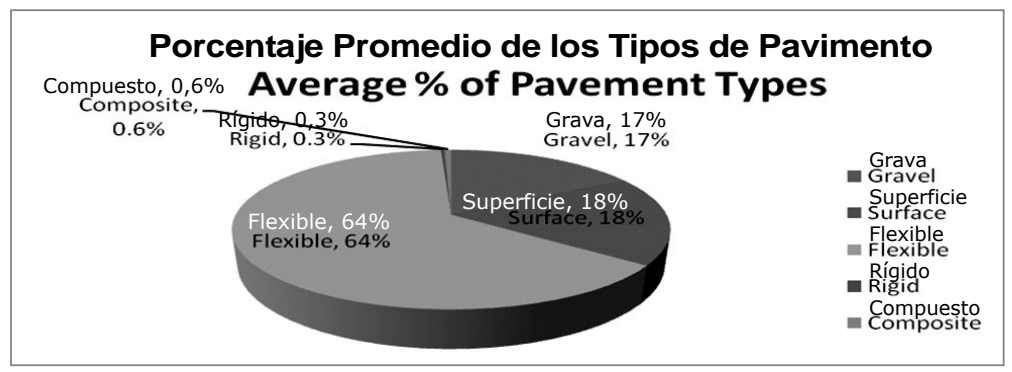

Figura 3. Resumen de los tipos de pavimento por los organismos federales, provinciales y territoriales Figure 3. Summary of pavement types for federal/provincial/territorial agencies 


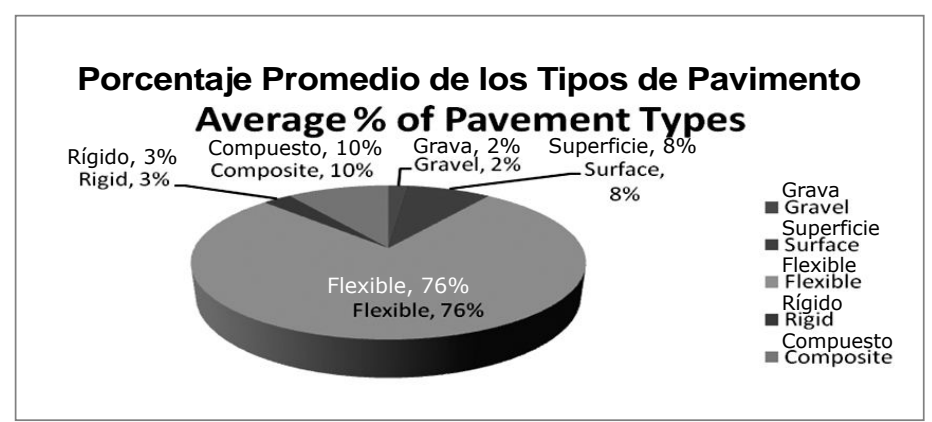

Figura 4. Resumen de los tipos de pavimento por las municipalidades encuestadas Figure 4. Summary of pavement types for cities surveyed

Table 2. ¿Qué plazo o período de planificación utiliza? Table 2. What planning horizons or periods do you use?

\begin{tabular}{||c|c|c|c|}
\hline $\begin{array}{c}\text { Período } \\
\text { Period }\end{array}$ & $\begin{array}{c}\text { Municipalidades } \\
\text { Cities }\end{array}$ & Provincial/Federal/Territorial & $\begin{array}{c}\text { Otros } \\
\text { Others }\end{array}$ \\
\hline 1-2 años/years & $7 \%$ & $8 \%$ & \\
\hline $1-5$ años/years & $7 \%$ & $70 \%$ & $50 \%$ \\
\hline $3-5$ años/years & $60 \%$ & & \\
\hline $5-10$ años/years & $33 \%$ & $\mathbf{8} \%$ & \\
\hline $1-10$ años/years & & $15 \%$ & $50 \%$ \\
\hline+10 años/years & & & \\
\hline
\end{tabular}

*Nota: Los porcentajes pueden no ser igual al $100 \%$ por redondeo o respuestas múltiples *Note: Percentages may not add up to $100 \%$ due to rounding and or multiple responses

Pavement and Asset Data Requirements, Collection Methods and Database.

Tabla 3A. Su base de datos para pavimentos ise encuentra integrada a un sistema de gestión de activos? Table 3A. Is your pavement database integrated with an asset management system?

\begin{tabular}{|c|c|c|c||}
\hline Respuesta/Answer & Municipalidades/Cities & Provincial/Federal/Territorial & Otros/Others \\
\hline No & $\mathbf{4 0} \%$ & $\mathbf{6 9} \%$ & $33 \%$ \\
\hline Si/Yes & $60 \%$ & $31 \%$ & $22 \%$ \\
\hline no disp./n/a & & & \\
\hline Sin respuesta/No answer & & & $44 \%$ \\
\hline
\end{tabular}

Tabla 3B. En caso de respuesta negativa, ¿tiene planificado hacerlo dentro los próximos 5 años? Table 3B. If no, Do you plan to do so in the next 5 years?

\begin{tabular}{|c|c|c|c||}
\hline Respuesta/Answer & Municipalidades/Cities & Provincial/Federal/Territorial & Otros/Others \\
\hline No & & $\mathbf{1 5 \%}$ & $\mathbf{2 2} \%$ \\
\hline Si/Yes & $\mathbf{4 0} \%$ & $\mathbf{5 4 \%}$ & $\mathbf{1 1 \%}$ \\
\hline no disp./n/a & $\mathbf{6 0} \%$ & $31 \%$ & $\mathbf{2 2} \%$ \\
\hline Sin respuesta/No answer & & & $44 \%$ \\
\hline \hline
\end{tabular}


Tabla 4. ¿Qué sistema de referencia de ubicación usa normalmente su organización?

Table 4 . What is the location referencing system your agency currently uses?

\begin{tabular}{||c|c|c|c||}
\hline Sistema/System & Municipalidades/Cities & Provincial/Federal/Territorial & Otros/Others \\
\hline Ramal-secciones/Branch-section & $53 \%$ & $15 \%$ & \\
\hline Base coordinada/Coordinate based & $20 \%$ & & $11 \%$ \\
\hline Nodo-enlace/Node-link & $27 \%$ & $15 \%$ & $11 \%$ \\
\hline Marca ruta-km (poste)/Route-km post & & $\mathbf{6 2} \%$ & $\mathbf{4 4} \%$ \\
\hline No disp./n/a & & $\mathbf{1 1} \%$ \\
\hline Sin resp./No answer & & $\mathbf{8 2} \%$ \\
\hline \hline
\end{tabular}

Tabla 5. ¿Su base de datos forma parte de una GIS (Sistema de Información Geográfica) que incorpora, por ejemplo, los servicios subterráneos (donde sea aplicable), otras características de los caminos, etc.?

Table 5. Is your database part of a GIS that incorporates, for example, underground services (where applicable), other road features etc.?

\begin{tabular}{|c|c|c|c||}
\hline Respuesta/Answer & Municipalidades/Cities & Provincial/Federal/Territorial & Otros/Others \\
\hline No & $\mathbf{2 0} \%$ & $\mathbf{5 4 \%}$ & $\mathbf{4 4 \%}$ \\
\hline Si/Yes & $\mathbf{8 0} \%$ & $\mathbf{4 6 \%}$ & $\mathbf{1 1 \%}$ \\
\hline no disp./n/a & & & $\mathbf{1 1} \%$ \\
\hline Sin respuesta/No answer & & & $33 \%$ \\
\hline
\end{tabular}

Tabla 6. ¿Su base de datos se adapta a la segmentación dinámica?

Table 6. Does your database accommodate dynamic segmentation?

\begin{tabular}{|c|c|c|c||}
\hline Respuesta/Answer & Municipalidades/Cities & Provincial/Federal/Territorial & Otros/Others \\
\hline No & $\mathbf{6 0} \%$ & $\mathbf{3 8} \%$ & $\mathbf{3 3} \%$ \\
\hline $\mathrm{Si} /$ Yes & $\mathbf{4 0} \%$ & $\mathbf{5 4} \%$ & $\mathbf{2 2} \%$ \\
\hline no disp./n/a & & $\mathbf{8} \%$ & \\
\hline Sin respuesta/No answer & & & $\mathbf{4 4} \%$ \\
\hline
\end{tabular}

Do you use the following?

Tabla 7A. Índice International de Rugosidad (IRI)

Table 7A. International Roughness Index

\begin{tabular}{|c|c|c|c||}
\hline Respuesta/Answer & Municipalidades/Cities & Provincial/Federal/Territorial & Otros/Others \\
\hline No & $\mathbf{3 3} \%$ & $\mathbf{1 5 \%}$ & $\mathbf{2 2} \%$ \\
\hline Si/Yes & $\mathbf{6 0} \%$ & $\mathbf{8 5} \%$ & $\mathbf{6 7 \%}$ \\
\hline no disp./n/a & & & \\
\hline Sin respuesta/No answer & $7 \%$ & & $\mathbf{1 1 \%}$ \\
\hline
\end{tabular}

Tabla 7B. Índice de Adecuación Estructural (SAI)

Table 7B. Structural Adequacy Index

\begin{tabular}{||c|c|c|c||}
\hline Respuesta/Answer & Municipalidades/Cities & Provincial/Federal/Territorial & Otros/Others \\
\hline No & $40 \%$ & $62 \%$ & $44 \%$ \\
\hline Si/Yes & $47 \%$ & $31 \%$ & $33 \%$ \\
\hline no disp./n/a & $13 \%$ & $8 \%$ & $22 \%$ \\
\hline Sin respuesta/No answer & & & \\
\hline
\end{tabular}


Tabla 7C. Respuesta al tipo de escala que usan los organismos para el Índice de Adecuación Estructural Table 7C. Response to the type of scale the agencies use for the Structural Adequacy Index

\begin{tabular}{||c|c|c|c||}
\hline Escala/Scale & Municipalidades/Cities & Provincial/Federal/Territorial & Otros/Others \\
\hline $0-10$ & $13 \%$ & $23 \%$ & \\
\hline $0-100$ & $13 \%$ & & $22 \%$ \\
\hline no disp./n/a & $47 \%$ & $69 \%$ & $11 \%$ \\
\hline Sin respuesta/No answer & $20 \%$ & $8 \%$ & $67 \%$ \\
\hline
\end{tabular}

Tabla 7D. Índice de Deterioro Superficial (SDI)

Table 7D. Surface Distress Index

\begin{tabular}{||c|c|c|c||}
\hline Respuesta/Answer & Municipalidades/Cities & Provincial/Federal/Territorial & Otros/Others \\
\hline No & $\mathbf{2 0} \%$ & $\mathbf{2 3} \%$ & $\mathbf{1 1} \%$ \\
\hline Si/Yes & $73 \%$ & $\mathbf{6 9} \%$ & $\mathbf{5 6 \%}$ \\
\hline no disp./n/a & $7 \%$ & $\mathbf{8} \%$ & \\
\hline Sin respuesta/No answer & & $33 \%$ \\
\hline
\end{tabular}

Tabla 7E. Respuesta al tipo de escala que usan los organismos para el Índice de Deterioro Superficial Table 7E. Response to the type of scale the agencies use for the Surface Distress Index

\begin{tabular}{|c|c|c|c||}
\hline Escala/Scale & Municipalidades/Cities & Provincial/Federal/Territorial & Otros/Others \\
\hline $\mathbf{0 - 1 0}$ & $\mathbf{3 3} \%$ & $\mathbf{4 6} \%$ & $\mathbf{2 2} \%$ \\
\hline $\mathbf{0 - 1 0 0}$ & $\mathbf{4 0} \%$ & $\mathbf{1 5 \%}$ & $\mathbf{1 1} \%$ \\
\hline $\mathbf{0 - 2 1 2}$ & & $\mathbf{8} \%$ & \\
\hline no disp./n/a & $\mathbf{2 7} \%$ & $\mathbf{3 1} \%$ & $\mathbf{1 1} \%$ \\
\hline Sin respuesta/No answer & & & $\mathbf{5 6} \%$ \\
\hline
\end{tabular}

Tabla 7F. Índice Compuesto (CI)

Table 7F. Composite Index

\begin{tabular}{|c|c|c|c|}
\hline Respuesta/Answer & Municipalidades/Cities & Provincial/Federal/Territorial & Otros/Others \\
\hline No & $13 \%$ & $31 \%$ & $22 \%$ \\
\hline Si/Yes & $60 \%$ & $62 \%$ & $11 \%$ \\
\hline no disp./n/a & & & \\
\hline Sin respuesta/No answer & $27 \%$ & $\mathbf{8} \%$ & $67 \%$ \\
\hline
\end{tabular}

Tabla 7G. Respuesta al tipo de escala que usan los organismos para su Índice Compuesto Table 7G. Response to the type of scale the agencies use for their Composite Index

\begin{tabular}{|c|c|c|c|}
\hline \hline Escala/Scale & Municipalidades/Cities & Provincial/Federal/Territorial & Otros/Others \\
\hline $0-10$ & $20 \%$ & $15 \%$ & \\
\hline $0-100$ & $27 \%$ & $46 \%$ & $11 \%$ \\
\hline $2-10$ & $7 \%$ & & \\
\hline $1-5$ & $7 \%$ & $38 \%$ & $11 \%$ \\
\hline no disp./n/a & $40 \%$ & & $78 \%$ \\
\hline Sin respuesta/No answer & \multicolumn{3}{|l}{} \\
\hline
\end{tabular}


Tabla 7H. Respuesta al tipo de Índice Compuesto usado por los organismos

Table 7H. Response to the type of Composite Index used by the agencies

\begin{tabular}{|c|c|c|c|}
\hline Compuesto/Composite & Municipalidades/Cities & Provincial/Federal/Territorial & Otros/Others \\
\hline PQI & $20 \%$ & $8 \%$ & \\
\hline RCI & $13 \%$ & & $11 \%$ \\
\hline SD & $7 \%$ & & \\
\hline IRI & & $8 \%$ & \\
\hline PCI & & $31 \%$ & \\
\hline SAI & $7 \%$ & & \\
\hline Pavement & $7 \%$ & & \\
\hline PCR & & $8 \%$ & \\
\hline $\mathrm{OCI}$ & $7 \%$ & & \\
\hline $\mathrm{BCl}$ & & $8 \%$ & \\
\hline SDI & $7 \%$ & & $11 \%$ \\
\hline PDI & & $8 \%$ & \\
\hline no disp./n/a & $40 \%$ & $38 \%$ & \\
\hline Sin respuesta/No answer & $13 \%$ & $8 \%$ & $78 \%$ \\
\hline
\end{tabular}

Tabla 8. ¿Realiza periódicamente encuestas sobre carga del eje?

Table 8. Do you conduct periodic axle load surveys?

\begin{tabular}{|c|c|c|c||}
\hline Respuesta/Answer & Municipalidades/Cities & Provincial/Federal/Territorial & Otros/Others \\
\hline No & $73 \%$ & $31 \%$ & $33 \%$ \\
\hline Si/Yes & $13 \%$ & $69 \%$ & $22 \%$ \\
\hline no disp./n/a & $13 \%$ & & \\
\hline Sin respuesta/No answer & & & $44 \%$ \\
\hline \hline
\end{tabular}

Tabla 9. ¿Incorpora Ud. específicamente cualquier dato relacionado con el medioambiente o clima en su base de datos de pavimentos y gestión de activos?

Table 9. Do you specifically incorporate any environment or climate related data in your pavement or asset management database?

\begin{tabular}{|c|c|c|c||}
\hline Respuesta/Answer & Municipalidades/Cities & Provincial/Federal/Territorial & Otros/Others \\
\hline No & $\mathbf{6 7} \%$ & $77 \%$ & $44 \%$ \\
\hline Si/Yes & $27 \%$ & $23 \%$ & $33 \%$ \\
\hline no disp./n/a & $7 \%$ & & \\
\hline Sin respuesta/No answer & & & $\mathbf{2 2} \%$ \\
\hline
\end{tabular}

Tabla 10. ¿Cómo gestiona o incorpora los datos de los costos de construcción o rehabilitación de los pavimentos en su Sistema de Gestión de Pavimentos (PMS) (por ej., recopilación anual de los costos unitarios o promedio, etc)? Table 10. How do you handle or incorporate cost data on pavement construction or rehabilitation within your PMS (i.e. annual compilation of average or benchmark unit costs, etc)?

\begin{tabular}{|c|c|c|c|}
\hline Datos de Costo/Cost Data & Municipalidades/Cities & Provincial/Federal/Territorial & Otros/Others \\
\hline Costo unitario/Unit Cost & $13 \%$ & $23 \%$ & \\
\hline Costo promedio/Average Cost & $13 \%$ & & \\
\hline Promedio/actividad/Average/activity & $7 \%$ & & \\
\hline Anual/Annual & $47 \%$ & $15 \%$ & \\
\hline Precios de licitación/Bidding prices & & $8 \%$ & \\
\hline Histórico/Historical & & $15 \%$ & \\
\hline Costo por $\mathrm{km} /$ Per $\mathrm{km}$ cost & $7 \%$ & $8 \%$ & \\
\hline \multicolumn{2}{|l|}{ Inflación post contrato/Post contract inflation } & $8 \%$ & \\
\hline Micropaver & & & $11 \%$ \\
\hline Revisión periódica/Periodic Review & $7 \%$ & & \\
\hline Rentabilidad/Economics & & & $11 \%$ \\
\hline CATS & & $8 \%$ & \\
\hline Contratos existentes/Existing Contracts & & & $11 \%$ \\
\hline no $\operatorname{disp} . / \mathbf{n} / \mathbf{a}$ & $7 \%$ & $15 \%$ & $11 \%$ \\
\hline Sin respuesta/No answer & & & $44 \%$ \\
\hline
\end{tabular}

*Nota: Los porcentajes pueden no ser igual al $100 \%$ ya que algunas organizaciones entregaron respuestas múltiples

* Note: Percentages may not add up to $100 \%$ as some organizations had multiple answers

*Nota: CATS = Construction Analysis and Tracking System, Análisis de construcción y sistema de seguimiento

**Note: CATS $=$ Construction Analysis and Tracking System 
Tabla 11. ¿Ha desarrollado su agencia un PMS a nivel de las redes viales? Table 11. Has your agency developed a network level PMS

\begin{tabular}{|c|c|c|c||}
\hline Respuesta/Answer & Municipalidades/Cities & Provincial/Federal/Territorial & Otros/Others \\
\hline No & $7 \%$ & $\mathbf{1 5 \%}$ & $\mathbf{1 1} \%$ \\
\hline Si/Yes & $\mathbf{9 3} \%$ & $\mathbf{8 5} \%$ & $\mathbf{4 4 \%}$ \\
\hline no disp./n/a & & & \\
\hline Sin respuesta/No answer & & & $\mathbf{4 4 \%}$ \\
\hline
\end{tabular}

Pavement Design (Structural and Economic Evaluation)

Tabla 12A. ¿Ha desarrollado su agencia un Manual de diseño de pavimentos? Table 12A. Does your agency have a Pavement Design Manual?

\begin{tabular}{|c|c|c|c||}
\hline Respuesta/Answer & Municipalidades/Cities & Provincial/Federal/Territorial & Otros/Others \\
\hline No & $\mathbf{6 4} \%$ & $\mathbf{5 4} \%$ & $\mathbf{6 4} \%$ \\
\hline Si/Yes & $\mathbf{3 6 \%}$ & $\mathbf{4 6 \%}$ & $\mathbf{2 7} \%$ \\
\hline no disp./n/a & & & \\
\hline Sin respuesta/No answer & & & $\mathbf{9 \%}$ \\
\hline
\end{tabular}

Tabla12B. Si la respuesta es si, ¿éste incluye un diseño para la rehabilitación? Table 12B. If yes, does it include rehabilitation design?

\begin{tabular}{|c|c|c|c||}
\hline Respuesta/Answer & Municipalidades/Cities & Provincial/Federal/Territorial & Otros/Others \\
\hline No & $\mathbf{2 9} \%$ & $\mathbf{1 5} \%$ & $\mathbf{1 8} \%$ \\
\hline Si/Yes & $\mathbf{2 9} \%$ & $\mathbf{4 6} \%$ & $\mathbf{3 6} \%$ \\
\hline no disp./n/a & $\mathbf{4 3} \%$ & $\mathbf{3 8} \%$ & $\mathbf{3 6} \%$ \\
\hline Sin respuesta/No answer & & & $\mathbf{9} \%$ \\
\hline
\end{tabular}

Tabla 12C. Si la respuesta es si, ¿éste incluye el mantenimiento? Table 12C. If yes, does it include maintenance?

\begin{tabular}{|c|c|c|c||}
\hline Respuesta/Answer & Municipalidades/Cities & Provincial/Federal/Territorial & Otros/Others \\
\hline No & $\mathbf{3 6} \%$ & $\mathbf{4 6} \%$ & $\mathbf{3 6} \%$ \\
\hline Si/Yes & $\mathbf{1 4} \%$ & $\mathbf{1 5} \%$ & $\mathbf{1 8} \%$ \\
\hline no disp./n/a & $\mathbf{5 0} \%$ & $\mathbf{3 8} \%$ & $\mathbf{3 6} \%$ \\
\hline Sin respuesta/No answer & & & $\mathbf{9} \%$ \\
\hline
\end{tabular}

Tabla 12D. Si la respuesta es si, ¿éste incluye la conservación? Table 12D. If yes, does it include preservation?

\begin{tabular}{|c|c|c|c||}
\hline Respuesta/Answer & Municipalidades/Cities & Provincial/Federal/Territorial & Otros/Others \\
\hline No & $\mathbf{3 6} \%$ & $\mathbf{3 8} \%$ & $\mathbf{2 7} \%$ \\
\hline Si/Yes & $\mathbf{1 4} \%$ & $\mathbf{2 3} \%$ & $\mathbf{2 7} \%$ \\
\hline no disp./n/a & $\mathbf{5 0} \%$ & $\mathbf{3 8} \%$ & $\mathbf{3 6} \%$ \\
\hline Sin respuesta/No answer & & & $\mathbf{9} \%$ \\
\hline
\end{tabular}

Tabla 12E. Si la respuesta es si, ¿éste incluye un diseño para drenajes? Table 12E. If yes, does it include drainage design?

\begin{tabular}{|c|c|c|c||}
\hline Respuesta/Answer & Municipalidades/Cities & Provincial/Federal/Territorial & Otros/Others \\
\hline No & $\mathbf{2 9} \%$ & $\mathbf{3 1} \%$ & $\mathbf{2 7} \%$ \\
\hline Si/Yes & $\mathbf{2 1} \%$ & $\mathbf{3 1} \%$ & $\mathbf{2 7} \%$ \\
\hline no disp./n/a & $\mathbf{5 0} \%$ & $\mathbf{3 8} \%$ & $\mathbf{3 6} \%$ \\
\hline Sin respuesta/No answer & & & $\mathbf{9} \%$ \\
\hline
\end{tabular}


Implementation of a Pavement Management and Asset Management System.

Tabla 13. La gestión de los pavimentos se puede realizar operacionalmente en dos niveles básicos: redes y proyecto. Si Ud. ha implementado un sistema de gestión de los pavimentos, ¿éste considera los siguientes aspectos?: Table 13. Pavement management can be operationally carried out at two basic levels: network and project. If you have implemented a pavement management system, does it consider:

\begin{tabular}{|c|c|c|c|c|}
\hline & Respuesta/Answer & Municipalidades/Cities & Provincial/Federal/Territorial & Otros/Others \\
\hline Tipo/Type & No & $47 \%$ & $38 \%$ & $33 \%$ \\
\hline \multirow[t]{4}{*}{ Nivel del proyecto/Project level } & Si/Yes & & $15 \%$ & \\
\hline & no disp./n/a & $47 \%$ & $31 \%$ & $33 \%$ \\
\hline & Sin respuesta/No answer & $7 \%$ & $15 \%$ & $33 \%$ \\
\hline & No & $20 \%$ & $8 \%$ & $33 \%$ \\
\hline \multirow[t]{4}{*}{ Nivel de las redes viales/Network level } & Si/Yes & $40 \%$ & $62 \%$ & $22 \%$ \\
\hline & no disp./n/a & $33 \%$ & $15 \%$ & $22 \%$ \\
\hline & Sin respuesta/No answer & $7 \%$ & $15 \%$ & $22 \%$ \\
\hline & No & $20 \%$ & $23 \%$ & \\
\hline \multirow[t]{3}{*}{ Ambos/Both } & Si/Yes & $53 \%$ & $38 \%$ & $44 \%$ \\
\hline & no disp./n/a & $20 \%$ & $23 \%$ & $22 \%$ \\
\hline & Sin respuesta/No answer & $7 \%$ & $15 \%$ & $33 \%$ \\
\hline
\end{tabular}

Tabla 14. ¿Tiene planificado hacer cambios mayores en su PMS?

Table 14. Are you planning any major changes in your PMS?

\begin{tabular}{|c|c|c|c||}
\hline Respuesta/Answer & Municipalidades/Cities & Provincial/Federal/Territorial & Otros/Others \\
\hline No & $13 \%$ & $31 \%$ & $11 \%$ \\
\hline Si/Yes & $\mathbf{8 0} \%$ & $46 \%$ & $44 \%$ \\
\hline no disp./n/a & $7 \%$ & $23 \%$ & $22 \%$ \\
\hline Sin respuesta/No answer & & & $22 \%$ \\
\hline
\end{tabular}

Tabla 15. ¿Su PMS opera a nivel estratégico para elaborar una lista presupuestaria consolidada para todos los activos? Table 15. Does it operate at the strategic level to produce one consolidated budget list for all assets?

\begin{tabular}{|c|c|c|c||}
\hline Respuesta/Answer & Municipalidades/Cities & Provincial/Federal/Territorial & Otros/Others \\
\hline No & $73 \%$ & $\mathbf{4 6} \%$ & $\mathbf{1 1} \%$ \\
\hline Si/Yes & $7 \%$ & $\mathbf{2 3} \%$ & $\mathbf{1 1 \%}$ \\
\hline no disp./n/a & $\mathbf{1 4 \%}$ & $\mathbf{3 1} \%$ & $\mathbf{2 2} \%$ \\
\hline Sin respuesta/No answer & $7 \%$ & & $\mathbf{5 6} \%$ \\
\hline
\end{tabular}

Tabla 16. Los sistemas de gestión ¿alimentan el Sistema de gestión de los activos (AMS)? Table16. Do all management systems feed into the AMS?

\begin{tabular}{|c|c|c|c||}
\hline Respuesta/Answer & Municipalidades/Cities & Provincial/Federal/Territorial & Otros/Others \\
\hline No & $\mathbf{8 0} \%$ & $\mathbf{5 4 \%}$ & $\mathbf{1 1} \%$ \\
\hline $\mathrm{Si} /$ Yes & & $\mathbf{1 5 \%}$ & $\mathbf{1 1 \%}$ \\
\hline no disp./n/a & $\mathbf{7 \%}$ & $\mathbf{3 1} \%$ & $\mathbf{2 2} \%$ \\
\hline Sin respuesta/No answer & $\mathbf{1 4 \%}$ & & $\mathbf{5 6} \%$ \\
\hline
\end{tabular}


6.2 Issues, Opportunities and Future Prospects This section is directly from the 2011 survey and the results are consolidated into the following list.

Describe what consultants or researchers could do to assist with tools your agency is lacking:

- Provide support in developing long-term performance specifications and design for pavements

- Asset Management System, including right-of-way asset management and underground utilities

- Focus research in defining the "sweet spots" on the deterioration curve which optimizes the timing of various preventative or rehabilitative treatments

- Issues related to funding constraints, and lack of resources for people and project follow-up, and loss of in-house expertise

- High speed and high quality data collection techniques relating to pavement distress and deflection

- Pavement design issues such as dependable backcalculation method/software and development of mechanistic design protocols to address use of non-traditional construction materials (e.g. cement stabilized granular, cold-in-place, hot-in-place, etc.)

- Methods to detect frost problem (discrimination between surface and deep frost problem), profile survey in winter and classification and frost measurement of frost cracking.

Any items that should be included in the new 2011 PADMG:

- Municipal constraints (e.g. curb and gutter, utilities) and a holistic management approach

- High speed and automated data collection such as IRI for QA, cracking data that has been collected using automated means

- Monitoring and evaluation procedures of rehabilitation/recycling techniques

- Structural characterization for Asset Management inputs to augment current inputs 
- Pavement design related issues such as transition from dynaflect to FWD, implementation of the MEPDG into pavement design, mechanistic designs and protocols and also how to implement structural characterization into a strategic and tactical asset management models and life cycle costing

- Environmentally sustainable and more environmentally friendly road materials and systems such as a green pavement rating system, more usage of RAP, warm mix and half-warm mix asphalts

- Performance based on remaining life, life cycle economic evaluation and investment strategies, optimization of rehabilitation budget to maximize the enhancement of the network quality, and best practices to narrow down differences between the network level and project level analysis

- Practical aspects of PMS implementation

- Innovative recycling methods

- Thin surfacing, micro-surfacing

- Trench cut restoration issues and at intersections on downhill grades

\section{Summary and comparison of the 1993 and 2010 surveys}

The following summarizes and compares the 1993 and 2010 survey responses. Although responses were also received from non-road authorities, the responses below are from road owners only and exclude public private partnerships.

\subsection{General Information}

Planning horizons range from 1 to 10 years with most using 1 to 5 years in 1993 and 3 to 5 years for the 2010 survey. Rehabilitation, maintenance and preservation dominate pavement expenditures. 
Tabla 17. Resumen de los porcentajes gastados en rehabilitación, mantenimiento y conservación

Table 17. Summary of the percentages spent on rehabilitation, maintenance and preservation

\begin{tabular}{||c|c|c|c||}
\hline \multicolumn{2}{|c|}{ Encuesta de 1993/1993 Survey } & \multicolumn{2}{c||}{ Encuesta de 2010/2010 Survey } \\
\hline Provincias y Territorios/Provinces and Territories & Hasta un $80 \% /$ Up to $80 \%$ & $\begin{array}{c}\text { Organismos Provinciales/Federales/Territoriales } \\
\text { Provincial/Federal/Territorial Agencies }\end{array}$ & $68 \%$ \\
\hline Federales/Federal & Hasta un 65\%/Up to 65\% & Ayuntamientos/Municipalidades/Cities/Municipalities & $66 \%$ \\
\hline Municipalidades/Municipalities & Hasta un 95\%/Up to 95\% & & \\
\hline
\end{tabular}

\subsection{Pavement and Asset Data Requirements, Collection Methods and Database}

\begin{tabular}{|c|c|c|}
\hline & 1993 & 2010 \\
\hline $\begin{array}{l}\text { - Organismos con bases de datos integradas de caminos y pavimentos } \\
\text { - Agencies with integrated road and pavement data bases }\end{array}$ & no/disponible/n/a & $35 \%$ \\
\hline $\begin{array}{l}\text { - Organismos con base de datos integrada con el Sistema de Gestión de Activos (AMS) } \\
\text { - Agencies with pavement database integrated with Asset Management Systems (AMS) }\end{array}$ & no/disponible/n/a & $38 \%$ \\
\hline $\begin{array}{l}\text { - Organismos con GIS como parte de la base de datos } \\
\text { - Agencies with GIS as part of database }\end{array}$ & $35 \%$ & $74 \%$ \\
\hline $\begin{array}{l}\text { - Organismos donde la base de datos se ajusta al seccionamiento dinámico } \\
\text { - Agencies where database accommodates dynamic sectioning } \\
\text { - Organismos que periódicamente recopilan } \\
\text { - Agencies that periodically collect }\end{array}$ & $65 \%$ & $39 \%$ \\
\hline o Datos de deflexión/Deflection data & $70 \%$ & $72 \%$ \\
\hline o Datos de rugosidad/Roughness data & $70 \%$ & $71 \%$ \\
\hline o Datos de deterioro superficial/Surface distress data & $90 \%$ & $66 \%$ \\
\hline $\begin{array}{l}\text { o Datos de derrapado/Skid data } \\
\text { - Principales dispositivos de medición } \\
\text { - Main measurement devices }\end{array}$ & $10 \%$ & $16 \%$ \\
\hline
\end{tabular}

Tabla 18. Resumen de los principales dispositivos de medición usados por los organismos Table 18. Summary of the main measurement devices used by agencies

\begin{tabular}{||c|c|c|c||}
\hline \multicolumn{2}{|c|}{ Encuesta de 1993/1993 Survey } & \multicolumn{2}{c|}{ Encuesta de 2010/2010 Survey } \\
\hline Deflexión/Deflection & Benkleman, Beam, Dynaflect, FWD & Deflexión/Deflection & Benkleman, Beam, Dynaflect, FWD \\
\hline Rugosidad/Roughness & Road Meters (Cox, Mays) ARAN & Rugosidad/Roughness & Automatizado/Automated \\
\hline Deterioro superficial/Surface Distres\$ & Manual/Visual & Deterioro superficial/Surface Distress & $\begin{array}{c}\text { Manual, Semi-automatizado, automatizado/ } \\
\text { Manual, Semi-automated, automated }\end{array}$ \\
\hline Derrapado/Skid & ASTM E274, SCRIM, BPN, Mu-Meter & Derrapado/Skid & $\begin{array}{c}\text { No se entregaron respuestas/ } \\
\text { No answers provided }\end{array}$ \\
& & & \\
\hline
\end{tabular}

-Uso de índices por organismos/Use of indices by agencies

o Índice de comodidad en el rodado/Riding Comfort Index o Índice de adecuación estructural/Structural Adequacy Index o Índice de deterioro superficial/Surface Distress Index o Índice compuesto/Composites Index

- Procedimientos para el desarrollo de modelos de predicción del comportamiento

- Procedures for developing performance prediction models o Regresión/Regression o Opinión de expertos/Expert Opinion o Markov o Bayesian/Markov or Bayesian o Otro/Other

- Organismos que recopilan periódicamente mediciones de carga de los ejes - Agencies that collect periodic axle load surveys

\begin{tabular}{ll} 
& \\
\hline & \\
$40 \%$ & $46 \%$ \\
$25 \%$ & $37 \%$ \\
$40 \%$ & $66 \%$ \\
$30 \%$ & $44 \%$ \\
& \\
& \\
$40 \%$ & $36 \%$ \\
$20 \%$ & $31 \%$ \\
$15 \%$ & $0 \%$ \\
$0 \%$ & $22 \%$ \\
$25 \%$ & $35 \%$
\end{tabular}




\subsection{Network level needs analysis and priority} programming

- Organismos que usan el análisis del costo del ciclo de vida en el análisis de rehabilitación

- Agencies using life-cycle cost analysis in rehabilitation analysis

- Organismos que consideran los costos del usuario en el análisis económico

\begin{tabular}{ll}
\hline $50 \%$ & $66 \%$ \\
$30 \%$ & $42 \%$ \\
$1-5$ yrs & $1-5$ yrs \\
$75 \%$ & $58 \%$
\end{tabular}

- Periodo programático para rehabilitaciones (en general)

- Program period for rehabilitation (generally)

- Organismos que separan sus programas de mantenimiento y rehabilitación

- Agencies that separate their maintenance and rehabilitation programs

- Enfoques de priorización

- Prioritizing approaches used

o Clasificación/Ranking
o Apreciación/Judgement
o Combinación de clasificación y apreciación/Combination of ranking and judgement
o Costo marginal-efectividad/Marginal Cost-Effectiveness
o Árbol de decisiones/Decision Trees
o Optimización/Optimization
o Otro/Other

$\begin{array}{ll}20 \% & 6 \% \\ 15 \% & 8 \% \\ 30 \% & 0 \% \\ 20 \% & 17 \% \\ 0 \% & 3 \% \\ 0 \% & 14 \% \\ 0 \% & 17 \%\end{array}$

*Nota - Otro incluye: Análisis del tipo de contabilidad del valor de la red vial, vida útil remanente descontada, listas presentadas por las regiones/distritos y plan de inversión a 20 años plazo como punto inicial, luego incorpora otros planes de departamentos, ajustes para restricciones de recursos financieros y activador del Índice de Calidad del Pavimento (PQI).

*Note other includes: Accounting type analysis of network value, discounted remaining life, lists submitted from regions/districts and 20 year capital plan as start point then incorporate other department plans then adjust for funding constraints, and PQI trigger

7.4 Implementation of a Pavement Management and Asset Management System

- Organismos que utilizaron las pautas de implementación de la Guía de 1977

- Agencies who used implementation guidelines from the 1977 guide

- Organismos que utilizaron las pautas de implementación de la Guía de 1997

$\mathbf{0} \%$

- Agencies who used implementation guidelines from the 1997 guide

- Nivel en que los organismos operan sus PMS

- Level at which agencies operate their PMS

o Nivel de redes viales únicamente/Network level only

o Nivel de proyecto únicamente/Project level only

o Ambos niveles/Both levels

- Organismos que planifican cambios mayores en sus PMS

- Agencies planning major changes in their PMS

- Ejemplo de ítemes para cambios mayores en el PMS como se describe en la Tabla 19

- Examples of items for major changes to PMS as shown in Table 19

Tabla 19. Resumen comparativo de ejemplos de ítemes para cambios mayores en el PMS

Table19. Summary comparison of examples of items for major changes in PMS

\begin{tabular}{|c|c|}
\hline Encuesta de 1993/1993 Survey & Encuesta de 2010/2010 Survey \\
\hline $\begin{array}{l}\text { o Condición vs. año del modelo/Condition vs. age models } \\
\text { o Sistema total integrado/Total integrated system } \\
\text { o Sistema experto para la estimación del deterioro/Expert system for estimating } \\
\text { deterioration } \\
\text { o Desarrollo de estrategia M\&R/M\&R strategy development } \\
\text { o Integración de PMS con GIS/Integration of PMS with GIS } \\
\text { o Datos de costo del tratamiento de mantenimiento/Maintenance treatment } \\
\text { cost data } \\
\text { o Análisis de prioridad mejoradalmproved priority analysis } \\
\text { o Más medidas objetivas/More objective measures }\end{array}$ & $\begin{array}{l}\text { o Ensayo de deflexión (FWD) para arterias viales/Deflection testing (FWD) } \\
\text { for arterial roads } \\
\text { o Integración de los datos FWD con datos GPR/Integration of FWD data } \\
\text { and GPR data } \\
\text { o Incremento de la capacidad al sistema/Increase capacity to system } \\
\text { o Posible actualización del PMS/Possibly updating PMS } \\
\text { o Coordinación con sistema de mantenimiento computarizado/Coordinate } \\
\text { with computerized maintenance management system } \\
\text { o Cambio de } \mathrm{RCI} \text { a IRI/Change from RCI to IRI } \\
\text { o Consideración del modelo de rehabilitación del deterioro/Consideration } \\
\text { for rehabilitation deterioration modeling }\end{array}$ \\
\hline
\end{tabular}


- Organismos que indican lo siguiente como aspectos de alta prioridad:

- Agencies indicating the following as high priority issues:

o Deterioro de redes viales pavimentadas/Deterioration of paved network

o Futuro incremento en límites de carga/Future increases in load limits

\begin{tabular}{ll} 
o Uso de productos de desecho y reciclaje futuro/Use of waste products and future recycling65\% & $63 \%$ \\
\hline
\end{tabular}

$\mathbf{8 5} \% \quad \mathbf{8 0} \%$

o Estricta regulación de salud medio ambiental/Strict environmental and health regulations $60 \% \quad 68 \%$

o Vapores de la pavimentación con asfalto/Fumes from asphalt paving $\quad 60 \%$

$\begin{array}{ll}\text { o Disponibilidad de personal capacitado/Availability of skilled people } & 55 \% \\ \text { o Implementación de productos SHRP y C-SHRP/Implementing SHRP and C-SHRP products } & 50 \%\end{array}$

$61 \%$

$81 \%$

o Implementación de productos SHRP y C-SHRP/Implementing SHRP and C-SHRP products $\mathbf{5 0} \%$
o Privatización del mantenimiento de los pavimentos/Privatizing pavement maintenance $\quad \mathbf{4 0} \%$

$\mathbf{n} / \mathbf{d}$

$32 \%$

- Organismos que señalan lo siguiente como mayores oportunidades:

- Agencies indicating the following as major opportunities:

o Pavimentos de mayor duración y sitios ambientalmente más limpios

$65 \%$

$59 \%$

o Longer lasting pavements plus clean environment stance

o Conservación de los materiales/Conservation of materials

o Desarrollo de especificaciones basadas en comportamiento a largo plazo

o Development of long term performance based specifications

o Financiamiento estable para innovación/Stable financing for innovation

$60 \%$

$60 \% \quad 66 \%$

$60 \% \quad 39 \%$

$50 \% \quad 37 \%$

\section{Conclusion and future steps}

This paper describes a large initiative that is currently underway to develop the new 2011 PADMG. This paper has presented a brief overview of the new 2011 PADMG and the findings from both the 1993 and 2010 surveys. Some of the detailed findings from the 2010 survey have been presented. These findings are intended to be highlights of the more comprehensive survey summary.

Perhaps one of the most surprising findings from the 2010 survey is in fact that despite a maturing road network, a large percentage of agency budgets seems to be allocated to new construction. Another notable change is that agencies are using slightly longer planning horizons in 2010 and are using PMS more extensively as compared to 1993. Several other smaller differences were noted between 1993 and 2010 and appear to primarily relate to changes in experience, expertise and the evolution of pavement design and management practices. With respect to issues opportunities and future prospects, generally the high priority areas remain unchanged from 1993 to 2010 with a greater emphasis in 2010 on the environment and the availability (or lack thereof) of skilled people. 
The survey was very important for determining what information should be included in the design guide. It also provided valuable insight into general practices across Canada. The TAC Asset Management Task Force and a performance measures Steering Group has used the survey results for various inititives. Finally, the new 2011 Guide promises to be a valuable tool for practitioners, managers and educators both nationally and internationally.

\section{Acknowledgements}

The authors of this paper gratefully acknowledge the contributions of all the members of the consortium and the members of the Project Steering Committee. The efforts of Laura Bland, Administrative Assistant, at the University of Waterloo and the staff members at TAC are also appreciated.

\section{Referencias / References}

Tighe S. (2009a), “Development of a National Pavement Asset Design and Management Guide (PADMG), Canada Consortium Proposal", Transportation Association of Canada.

Tighe S. (2009b), "Inventory Survey of Current Practices for Use in Preparing Transportation Association of Canada's New: Pavement Asset Design and Management Guide (PADMG)", Transportation Association of Canada. Tighe S., Bland L., (2010a), "Pavement Asset Design and Management Guide, State-of-the-Practice Survey Summary". Tighe S., Juhasz M., (2010a), "Canadian Pavement Design and Management Current State-of-the-Practice", Transportation Association of Canada Annual Conference, Halifax, Nova Scotia, Septiembre 2010. 